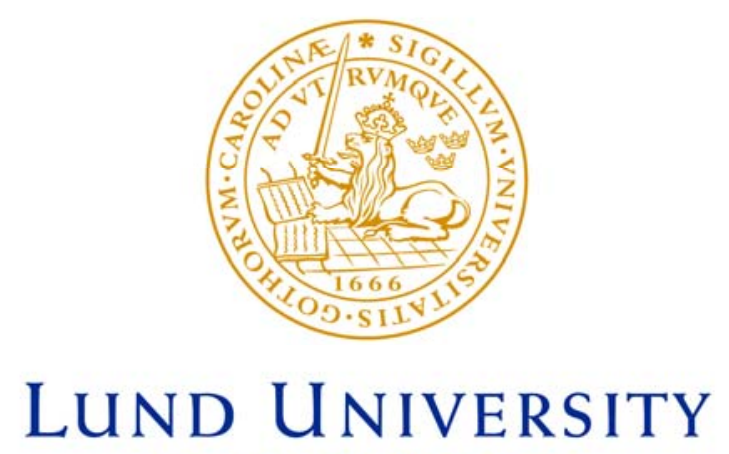

Faculty of Medicine

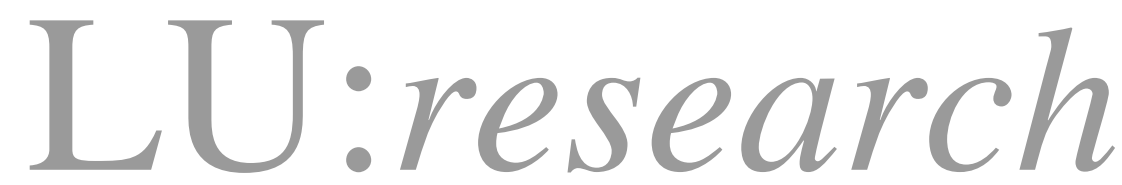

Institutional Repository of Lund University

This is an author produced version of a paper published in Diabetes Research and Clinical Practice. This paper has been peer-reviewed but does not include the final publisher proof-corrections or journal pagination.

Citation for the published paper:

Lovestam-Adrian, Monica and Hansson-Lundblad,

Catarina and Torffvit, Ole.

"Sight-threatening retinopathy is associated with lower mortality in type 2 diabetic subjects:

A 10-year observation study"

Diabetes Research and Clinical Practice, 2006, Issue: Dec 16.

http://dx.doi.org/10.1016/j.diabres.2006.10.026

Access to the published version may require journal subscription. Published with permission from: Elsevier 


\section{SIGHT-THREATENING RETINOPATHY IS ASSOCIATED WITH LOWER MORTALITY IN TYPE 2 DIABETIC SUBJECTS: A 10-YEAR OBSERVATION STUDY.}

Monica Lövestam-Adrian $\mathrm{PhD}^{1}$, Catarina Hansson-Lundblad ${ }^{1}$, Ole Torffvit $\mathrm{Phd}^{1}$

From the Departments of Clinical Sciences in Lund ${ }^{1}$, Lund University, Sweden

Correspondence:

Dr. Monica Lövestam-Adrian

Department of Ophthalmology

University Hospital, Lund

SE-221 85 Lund

Sweden

e-mail: monica.lovestam_adrian@med.lu.se

Fax: 46/46/172721 


\section{Abstract}

Aims: To study associations between diabetic retinopathy and development of stroke, myocardial infarction and death in type 2 diabetic patients.

Methods: During a 10-year observation period, 363 type 2 diabetic patients (diagnosis $\geq 30$ years of age) attending an outpatient clinic were studied regarding the prevalence and incidence of retinopathy and associated risk factors, i.e., $\left(\mathrm{HbA}_{1 \mathrm{c}}\right.$, blood pressure, albuminuria, plasma creatinine, age, sex and diabetes duration) in relation to the development of myocardial infarction, stroke and death. The degree of retinopathy was classified as no retinopathy, background or sight-threatening retinopathy, i.e., clinically significant macular edema, severe non-proliferative or proliferative retinopathy.

Results: During the study period 62 patients had had myocardial infarction, 54 stroke and 99 patients died. Patients with sight-threatening retinopathy at baseline $(n=41)$ had a 2.2-fold increased $(\mathrm{P}<0.01)$ risk for death compared to patients with no or background retinopathy, even when controlled for medical risk factors. When adjusted for medical risk factors, patients with no retinopathy at baseline $(n=226)$ who remained without retinopathy or developed background retinopathy $(\mathrm{n}=187)$ during the study period, had a 3.6- fold increased risk for death (95\% CI, 1.1,11.8), $(\mathrm{P}=0.03)$, compared to patients who developed sightthreatening retinopathy $(n=39)$, while the incidence of myocardial infarction did not differ. More patients who developed sight-threatening retinopathy were treated with ACE inhibitors than patients who did not ( $41 \%$ vs. $24 \% ; \mathrm{P}=0.03)$.

Conclusion: Despite more medical risk factors, patients who developed sight-threatening retinopathy had lower mortality compared to patients with no or background retinopathy at follow-up. More patients who developed sight-threatening retinopathy were treated with ACE inhibitors but this seemed not to have influenced the lower mortality rate in this group, 
whereas the use of ACE inhibitors in patients who did not develop sight-threatening retinopathy was connected with lower mortality rate. 
Keywords: retinopathy; macrovascular complications; mortality; type 2 diabetes 


\section{Introduction}

Type 2 diabetes is associated with an increased cardiovascular mortality risk (Krolewski, Czyzyk, Janecko \& Kopczynski,1977;Fuller, Shipley, Rose, Jarrett \& Keen 1983; Moss, Klein \& Klein 1991). Strict metabolic and blood pressure control have shown to reduce the risk of microvascular complications (UK Prospective Diabetes Study Group 1998). The higher mortality rate in patients with late diabetic complications (Early Treatment Diabetes Study Group 2005) may reflect a common pathogenetic mechanism underlying micro and macrovascular complications. Diabetic retinopathy and visual impairment have been associated with increased mortality in diabetic subjects (Early Treatment Diabetes Study Group 2005; Davis, Hiller, Magli et al 1979; Klein, Klein \& Moss 1995). However, few studies (Klein, klein Scot, Moss \& Cruickshanks 1999) have examined the incidence and progression of retinopathy in relation to cardiovascular morbidity and all cause mortality during a longer follow-up period. The purpose of the present study was therefore to investigate whether development of retinopathy was associated with the incidence of myocardial infarction, stroke and death during a 10-year observation period in type 2 diabetic patients.

\section{Patients and Methods}

\section{Patients}

All type 2 diabetic patients (diabetes diagnosis $\geq 30$ years of age) regularly attending the Departments of Medicine and Ophthalmology, University Hospital, Lund, Sweden were included in a continuous 10- year follow-up study between 1986 and 1996. The medical examinations for all patients took place three times a year (median, range 2 to 4 times a year) and the ophthalmologic examinations at least every other year. The diagnosis of type 2 diabetes included only those who did not require insulin treatment within 2 years of diagnosis. 
Out of 451 type 2 diabetic patients initially studied (Torffvit\& Agardh 2000), 48 patients were excluded because of ketonuria at diabetes dignosis or because they were later admitted for ketoacidosis, 18 patients were lost for follow-up and data from the ophthalmological examination were not available in 22 patients. Of the remaining 363 patients (129 females) 74 were treated with oral drugs (sulfonylurea; $n=37$, metformin; $n=11$, or both ; $n=33$ ), and 287 with insulin (191 from baseline and an additional 96 patients received insulin during the study). Twenty patients were on both oral agents and insulin.

\section{Retinopathy}

Retinal examinations took place at least every other year. The classification of retinopathy was based on fundus photographys using a $45^{\circ}$ Topcon camera. Photography visualised three fields per eye; nasal, temporal and the macular region. In a few cases, where photographs were not available, the evaluation of retinopathy was based on a detailed description of retinopathy performed by an experienced ophthalmologist using biomicroscopy through a dilated pupil. Three retinopathy levels were used based on the worst affected eye: no retinopathy, background retinopathy, and sight-threatening retinopathy. Sight-threatening retinopathy included clinically significant macular oedema and/or severe non-proliferative retinopathy according to definitions by the ETDRS ( Early Treatment Diabetes Study Group 1985), or clinically significant macular oedema and/or proliferative retinopathy. All patients with sight-threatening retinopathy were treated with photocoagulation according to the guidelines from the ETDRS (Early Treatment Diabetes Study Group 1987) and the DRS (Diabetic Retinopathy Study Research Group 1981).

\section{Risk factors}

Age, diabetes duration, body mass index (BMI), $\mathrm{HbA}_{1 \mathrm{c}}$, blood pressure, urinary albumin, and serum creatinine levels were measured throughout the follow-up period. The mean logarithmic urinary-albumin, serum creatinine and the maximum of urinary albumin/ 
creatinine clearance ratio (U-ACCR) during the 10-year follow-up was calculated.

\section{Analytical techniques}

Glycosylated hemoglobin $\left(\mathrm{HbA}_{1 \mathrm{c}}\right)$ was analysed by ion-exchange chromatography (Bio-Rad, Richmond, CA) (1985-1987), by FPLC (Pharmacia, Uppsala, Sweden) (1988-1994) and by HPLC (1995-). The latter methods were adjusted to give similar results as the first one. Normal values was $4.0-5.3 \%$._Urinary albumin concentrations and urine- and serum creatinine levels were analysed as previously described (Lövestam-Adrian, Agardh \& Agardh 1999).. The UACCR was calculated as the ratio between the urinary albumin clearance and the urine creatinine clearance. Normal value is $<0.01 \times 10^{-3}$.

\section{Statistics}

ANOVA was used for multiple group comparisons of normally distributed continuous variables, and Bonferroni's test for post hoc analysis of multiple comparisons. Distributionfree data were analysed with Kruskal-Wallis test and then further analysed with Wilcoxon's sign-rank test (for paired data) or the Mann-Whitney $U$-test (for unpaired data). Chi-squared test $\left(x^{2}\right)$ was used for testing the difference of proportions between categorical variables. The association between heart disease, stroke or death and medical variables was investigated by the Cox regression analysis with forward stepwise selection. Retinopathy at entry and followup was included in the analysis as a variable consisting of three levels as defined above. Progression was tested by using the difference between retinopathy degree at baseline and at follow-up. The criteria for determining variables to be added to the model were based on the maximum partial likelihood estimate. In the case of vascular morbidity or death, the "survival time" was the time from baseline to the date of event or until follow-up. In the latter case it was censored. Relative risk for an event was taken from the Cox regression analysis. 


\section{Results}

\section{Patient characteristics at baseline}

At baseline 226 patients had no retinopathy, 96 background retinopathy and 41 patients sightthreatening retinopathy. Patients with sight-threatening retinopathy were older, had longer diabetes duration, higher $\mathrm{BMI}$, higher levels of mean $\mathrm{HbA}_{1 \mathrm{c}}$, systolic blood pressure, degree of albuminuria and U-ACCR compared to patients with no retinopathy, and higher BMI, degree of albuminuria and albumin creatinine clearance ratio compared to patients with background retinopathy. Patients with background retinopathy were older, had longer diabetes duration and higher systolic blood pressure compared to those with no retinopathy (Table 1).

\section{Baseline retinopathy and mortality}

The mortality rate was higher in patients with sight-threatening retinopathy and with sightthreatening and background retinopathy during the study period compared with patients with no retinopathy $(19 / 41$ vs. $51 / 226 ; p=0.001$, and $48 / 137$ vs. $51 / 226 ; p=0.01)$, respectively. No differences were seen in the incidence of myocardial infarction or stroke during the study period between the different levels of retinopathy.

Incidence of cardiovascular complications and death in relation to development of retinopathy in patients with no retinopathy at baseline.

The development of background retinopathy was $31 \%(71 / 226)$ and of sight-threatening retinopathy $17 \%(39 / 226)$, whereas the progression from background to sight-threatening retinopathy was 51\% (49/96). Patients who developed sight-threatening retinopathy more often had medical examinations than patients who did not, 5 (3-9) visits per year vs. 4 (0-9) visits per year, (median, range); $(\mathrm{p}=0.033)$, and had higher levels of mean $\mathrm{HbA}_{1 \mathrm{c}}$ and maximum level of albuminuria compared to both patients who did not develop retinopathy and those who developed background retinopathy, (Table 2). The incidence of myocardial infarction was higher in patients who did not develop any retinopathy compared to those with 
no retinopathy at baseline and who developed sight-threatening retinopathy (21/116 vs. 2/39; $\mathrm{p}=0.049)$. There was no difference regarding the incidence of stroke. Mortality rate was higher in patients who did not develop retinopathy compared to patients who developed sightthreatening retinopathy $(30 / 116$ vs. $3 / 39 ; \mathrm{p}=0.016)$ and higher in patients who developed background retinopathy compared to those who developed sight-threatening retinopathy $(18 / 71$ vs. $3 / 39 ; p=0.024)$. Patients who developed sight-threatening retinopathy had a longer follow-up time ( $9.9 \pm 1.0$ years) compared to patients who developed background retinopathy $(8.8 \pm 1.8$ years; $p=0.0369$ and patients with no retinopathy $(8.5 \pm 2.6$ years; $p=0.001)$ at follow-up.

Incidence of cardiovascular complications and death in relation to progression of retinopathy Patients who progressed from background to sight-threatening retinopathy had higher levels of mean $\mathrm{HbA}_{1 \mathrm{c}}$ levels $(\mathrm{p}=0.004)$ and of maximum albuminuria $(\mathrm{p}=0.041)$ compared to patients who did not progress. No difference in mortality rate was seen in patients with background retinopathy at baseline who continued to have background retinopathy (47/96) compared to those who developed sight-threatening retinopathy (49/96). 


\section{Relative risk -Cox regression analysis}

When adjusting for systemic risk factors, i.e., age, diabetes duration, $\mathrm{BMI}, \mathrm{HbA}_{1 \mathrm{c}}$, blood pressure, urinary albumin, and serum creatinine levels, patients with sight-threatening retinopathy at baseline had a 2.2 -fold $(95 \%$ confidence interval $(\mathrm{CI}), 1.3,3.6)$ increased risk ( $p=0.02)$ to die during the study compared to patients with no or background retinopathy, (Figure 1).

Patients with no retinopathy at baseline who still had no or background retinopathy at followup had a 3.6-fold (95\% CI, 1.1,11.8) increased risk for all-cause mortality $(\mathrm{p}=0.03)$ compared with patients who developed sight-threatening retinopathy, still when adjusting for medical risk markers, (Figure 2). Since the number of patients who died in the group who developed sight-threatening retinopathy was low, Fishers Exact Test was used as complement and showed that $48 / 187$ patients who did not develop retinopathy compared to $3 / 39$ patients who developed sight-threatening retinopathy died, $\mathrm{p}=0.012$.

\section{Underlying causes of death}

Cardiovascular complications were the cause of death in $51 \%$ of patients with no retinopathy, in $55 \%$ with background, and in $79 \%$ with sight-threatening retinopathy at baseline. For patients with no retinopathy at baseline, cardiovascular complications as an underlying cause of death in relation to retinopathy degree at follow-up were, $47 \%$ in patients with no retinopathy, $56 \%$ with background retinopathy and $67 \%$ in patients with sight-threatening retinopathy. In patients with no retinopathy at follow-up, cancer mortality and other causes (i.e. pneumonia and gastrointestinal haemorrhage) were the underlying causes of death in $50 \%$ of the patients. The different causes of mortality in relation to the development of retinopathy are shown in Table 3. 


\section{Antihypertensive treatment}

Forty-one percent of the patients who developed sight-threatening retinopathy had received treatment with ACE- inhibitors compared with $24 \%$ of the patients with no or background retinopathy at follow-up, $(\mathrm{p}=0.046)$. In a Kaplan -Meier analysis on overall survival time with incidence of sight -threatening retinopathy as factor and the use of ACE inhibitors as strata, treatment with ACE inhibitors was connected with a longer survival time in patients who did not develop sight - threatening retinopathy, $p=0.01$. The same relation could not be confirmed in patients who developed sight-threatening retinopathy. No differences were seen in the treatment with other antihypertensive drugs, i.e., Ca-blockers, ß-blockers and diuretics.

\section{Discussion}

In this 10 -year prospective study of type 2 diabetic patients, patients with background or sight-threatening retinopathy at baseline had an increased mortality compared to those without retinopathy, a finding supported by others Davis., Hiller, Magli et al 1979; Klein, Klein ,Klein\& Cruickshanks 1999). However, we also found that patients with no retinopathy at baseline who developed sight-threatening retinopathy during the study period had a reduced risk for death compared to those who did not.

Retinal micro vascular abnormalities have shown to be predictive for the incidence of coronary heart disease and stroke independent of diabetes and other risk factors (Wong, Klein Couper et al 2001; Wong, Klein, Sharrett et al 2002). In addition, more severe diabetic retinopathy has been associated with ischemic heart disease ( Fuller, Stevens\& Wang 2001; Ono, Kobayashi, Sasako et al. 2002). Diabetic retinopathy in type 2 diabetes is associated with diabetes duration, metabolic control and blood pressure (UK Prospective Diabetes Study Group 1998; Klein, Klein, Moss, Davis \& DeNets 1989). In the present study, patients with sight-threatening retinopathy at baseline had worse metabolic control, longer diabetes 
duration and signs of nephropathy compared to those with no or background retinopathy, which has been associated with mortality (Moss, Klein \& Klein 1991; Fuller, Stevens\& Wang 2001). In contrast to another study (Klein, Klein, Scot, Moss \& Cruickshanks 1999), we could not confirm a higher incidence of stroke in patients with severe retinopathy at baseline.

The results from the patients with no retinopathy at baseline with severe progression during the study period are a bit unexpected. Duration which is known to be a risk marker for both complications and death in diabetes was for certain longer in patients who developed background retinopathy but similar in patients who developed no or sight-threatening retinopathy. Thus the influence of duration on the results are not conclusive. Albuminuria has been reported to be an important risk marker for both cardiovascular events and death (Donnelly, Yeung \& Manning 2003), and patients who developed sight-threatening retinopathy from no retinopathy had at baseline higher levels of urinary albumin compared to patients who did not develop sight-threatening retinopathy. They had also worse metabolic control, but myocardial infarction and death were less frequent. Patients who developed sightthreatening retinopathy were medically examined more often, and one might speculate if that could have influenced the mortality from cancer and other causes. The underlying cause of mortality was cardiovascular events in $67 \%$ of patients with sight-threatening retinopathy compared to $47 \%$ in patients with no retinopathy. In patients without retinopathy the underlying causes of death were cancer and other causes in 50\%. However, the number of patients who died in the group who developed sight-threatening retinopathy was few, which set limits to the interpretations of the results from the Cox regression analysis. A Fisher's exact test could however confirm the tendency.

We acknowledge the limitation in our study, not having included lipids levels and data on current smoking. Furthermore, the follow-up time in the group who developed sightthreatening was longer and thus the patients in the other groups had less time to develop sight- 
threatening retinopathy which might bias the analysis. The effect of smoking on the incidence and progression of retinopathy vary between different studies. Some have reported no association (Owens, Volund, Jones et al 1988), while the UKPDS found smoking to be inversely related to the development of retinopathy (UK Prospective Diabetes Study Group50, 2001).

Blood pressure is an established risk marker for the development of retinopathy (UK Prospective Diabetes Study Group 69, 2004), and the UKPDS (United Kingdom Prospective Diabetes Study) showed that tight blood pressure control reduced both macrovascular and microvascular complications in hypertensive patients with type 2 diabetes (UK Prospective Diabetes Study Group 38, 1998). Intensified blood pressure therapy also has shown to lower the rate of mortality (Estacio, Jeffers, Gifford \& Schrier 2000). In the present study, the blood pressure was similar in patients who developed sight-threatening retinopathy and patients who did not, and almost equal to the blood pressure levels obtained in patients allocated to tight blood pressure control in the UKPDS (UK Prospective Diabetes Study Group 39, 1998), whereas the progression of retinopathy in our study was similar to those not assigned to tight blood pressure control in the UKPDS. The explanation might be the higher mean level of $\mathrm{HbA}_{1 \mathrm{c}}, 8.0-9.1 \%$, in our patients compared to $7.4 \%$ in patients with tight blood pressure control in the UKPDS with the limitations of slightly different analytical techniques for measuring $\mathrm{HbA}_{1 \mathrm{c}}$ levels.

The use of B-blockers was equal in patients who developed retinopathy and in those who did not, whereas more patients who developed sight- threatening from no retinopathy were treated with ACE inhibitors. However, when confirming the hypothesis, stratifying the data into the use of ACE inhibitors yes or no, only patients who did not develop sight-threatening retinopathy demonstrated any protection from the use of ACE inhibitors. The lack of connection in the group who developed sight-threatening retinopathy might partly be due to 
the low number of patients who died in the group. The protection of ACE inhibitors use in patients who did not develop sight-threatening retinopathy is supported by other studies, in which ACE inhibitors have been shown to reduce death rate, both in non-diabetic ( Garg \& Yusuf 1995) and diabetic patients (Yusuf, Lonn Bosch \& Gerstein 1999). In addition, in our Type 2 diabetic patients we have previously reported a lower cumulative hazard of myocardial infarction in patients treated with ACE inhibitors compared to patients treated with Ca-blockers and B-blockers (Torffvit \& Agardh 2001). Our study did not support any protective effect of ACE inhibitors on progression of retinopathy. This finding is in accordance with other studies, in which ACE inhibitors did not influence progression and development of retinopathy, (UK Prospective Diabetes Study Group 69, 2004; Pradhan, Fong, March, Jack et al, 2002), but in contrast to the EUCLID-study Chaturvedi, Sjolie, Stephenson, Abrahamian et al 1998). However, since many patients are treated with more than one antihypertensive drug, the present study can not be conclusive regarding the ACE-inhibitors effect.

In conclusion, type 2 diabetic patients with sight-threatening retinopathy at baseline had higher mortality rate whereas patients, who developed sight-threatening retinopathy had lower mortality, despite more medical risk markers, compared to patients with no- or background retinopathy at follow-up. More patients who developed sight-threatening retinopathy were treated with ACE inhibitors but this seemed not to have influenced the lower mortality rate in this group, whereas the use of ACE inhibitors in patients who did not develop sightthreatening retinopathy was connected with lower mortality rate.

\section{Acknowledgements}

This study was supported by grants from the Medical Faculty, Lund University, the Skane County Council Foundation for Research and Development, the Crown Princess 
Margareta's Committee for the Blind and the $2^{\text {nd }}$ O.N.C.E. international award for new technologies for the blind. 


\section{References}

1. N. Chaturvedi, A. Sjolie, JM. Stephenson, and the EUCLID Study Group, Effect of lisinopril on progression of retinopathy in normotensive people with type 1 diabetes. Lancet 351 (1998) 28-31.

2. M.D. Davis, R. Hiller, Y.L.M. Magli, et al. Prognosis for life in patients with diabetes: relation to severity of retinopathy. Trans Am Ophthalmol Soc 77 (1979) 144-170.

3. Diabetic Retinopathy Study Research Group, Photocoagulation treatment of proliferative diabetic retinopathy: clinical applications of Diabetic Retinopathy Study (DRS) findings: DRS report number 8. Ophthalmology 88 (1981) 583-600.

4. R. Donnelly, MJ. Yeung, G. Manning, Microalbuminuria: a common, independent cardiovascular risk factor, especially but not exclusively in type 2 diabetes. $\mathrm{J}$ Hypertens Supp 1 (2003) 7-12.

5. Early Treatment Diabetic Retinopathy Study Research Group. Associations of mortality and diabetes complications in patients with type 1 and type 2 diabetes: ETDRS report number 27. Diabetes Care 28 (2005) 617-625.

6. Early Treatment Diabetic Retinopathy Study Research Group. Photocoagulation for diabetic macular edema: ETDRS report number 1. Arch Ophthalmol 103 (1985) 17961806.

7. Early Treatment Diabetic Retinopathy Study Research Group. Photocoagulation for diabetic macular edema: ETDRS report number IV. Int Ophthalmol Clin 27 (1987) $265-272$.

8. R.O. Estacio, BW. Jeffers, N. Gifford, R.W. Schrier, Effect of blood pressure control on diabetic microvascular complications in patients with hypertension and type 2 diabetes. Diabetes Care 23 (2000) Suppl 2:B54-64. 
9. J. Fuller, L. Stevens, SL. Wang, the WHO Multinational study group. Risk factors for cardiovascular mortality and morbidity: The WHO multinational study of vascular disease in diabetes. Diabetologia (2001) (SUPP 2) S54-64.

10. J.H. Fuller, M.J. Shipley, G. Rose, R.J. Jarrett, H. Keen, Mortality from coronary heart disease and stroke in relation to degree of glycaemia: the Whitehall Study. BMJ 287 (1983) 867-870.

11. R. Garg, S. Yusuf, Overview of randomized trials of angiotensin-converting enzyme inhibitors on mortality and morbidity in patients with heart failure. Collaborative Group on ACE Inhibitor JAMA 273 (1995) 1450-56.

12. R. Klein, B.E.K. Klein, M.A. Moss, Age related eye disease and survival: the Beaver Dam Eye study. Arch Ophthalmol 113 (1995) 333-339.

13. R. Klein, B.E.K. Klein, S.E. Moss, M.D.Davis, D.L. DeNets. The Wisconsin Epidemiologic Study of Diabetic Retinopathy. IX. Four-year incidence and progression of diabetic retinopathy when age at diagnosis is 30 years or more. Arch ophthalmol 107 (1989) 244-49.

14. R. Klein, B.E.K. Klein, E. Scot, M.A. Moss, K.J. Cruickshanks, Association of ocular disease and mortality in a diabetic population. Arch Ophthalmol 117 (1999) 14871495.

15. A.S. Krolewski, A. Czyzy, D. Janeczko, J. Kopczynski, Mortality from cardiovascular disease among diabetics. Arch Ophthalmol 13 (1977) 345-350.

16. M. Lövestam-Adrian, E. Agardh, C.D. Agardh, The temporal development of retinopathy and nephropathy in type 1 diabetes mellitus during 15 years diabetes duration. Diab Res Clin Practice 45 (1999) 15-23.

17. S.E. Moss, R. Klein, B.E. Klein BE, Cause-specific mortality in a population-based study of diabetes. Am J Public Health 81 (1991) 1158-62. 
18. T. Ono, J. Kobayashi, Y. Sasako et al. The impact of diabetic retinopathy on long-term outcome following coronary artery bypass graft surgery. J Am Coll Cardiol 7: 40 (2002) 428-36.

19. D. Owens, A. Volund, D. Jones, et al: Reinopathy in newly presenting non-insulindependent (TypeII) diabetic patients. Diabetes Res 9 (1988) 59-65.

20. R. Pradhan, D. Fong, C. March, R. Jack, G. Rezapour, K. Norris, M.B. Davidson, Angiotensin-converting enzyme inhibition for the treatment of moderate to severe diabetic retinopathy in normotensive type 2 diabetic patients. A pilot study. J Diab Comp 16 (2002) 377-81.

21. O. Torffvit, C.D. Agardh, Treatment mode and development of diabetic complications. A 10-year observation study of 385 type 2 diabetic patients. Diabetes Research 35 (2000) 117-125.

22. O. Torffvit, C.D. Agardh, The impact of metabolic and blood pressure control on incidence and progression of nephropathy. A 10-year study of 385 type 2 diabetic patients. J Diab. Compl 15 (2001) 307-313.

23. U.K. Prospective Diabetes Study (UKPDS ) Group : Risk factors for incidence and progression of retinopathy in type II diabetes over 6 years from diagnosis, UKPDS 50. Diabetologia 44 (2001) 156-163.

24. U.K. Prospective Diabetes Study (UKPDS ) Group. Risks of progression of retinopathy and vision loss related to tight blood pressure control in type 2 diabetes mellitus, UKPDS 69. Arch Opthalmol 122 (2004) 1631-1640.

25. U.K. Prospective Diabetes Study (UKPDS ) Group. Tight blood pressure control and risk of macrovascular and microvascular complications in type 2 diabetes: UKPDS 38. BMJ 317 (1998) 703-13. 
26. U.K. Prospective Diabetes Study (UKPDS ) Group. Efficacy of atenolol and captopril in reducing risk of macrovascular and microvascular complications in type 2 diabetes: UKPDS 39. BMJ 317 (1998) 713-720.

27. UK Prospective Diabetes Study (UKPDS) Group. Intensive blood-glucose control with sulphonylureas or insulin compared with conventional treatment and risk of complications in patients with type 2 diabetes. Lancet 352 (1998) 837-853.

28. T.Y. Wong, R.Klein, D.J. Couper et al. Retinal microvascular abnormalities and incident strokes. The atherosclerosis risk in communities study. Lancet 358 (2001) $1134-40$.

29. T.Y. Wong, R. Klein, A.R. Sharrett, et al. Retinal arteriolar narrowing and incident coronary heart disease in men and women. The atherosclerosis risk in communities study. JAMA 287 (2002) 1153-9.

30. S. Yusuf, E. Lonn, J. Bosch, H.Gerstein, Summary of randomized trials of angiotensin converting enzyme inhibitors. Clin Exp Hypertens 21 ( 1999) 835-45. 
Table 1. Patient characteristics at baseline

\begin{tabular}{|c|c|c|c|}
\hline & No retinopathy & $\begin{array}{l}\text { Background } \\
\text { retinopathy }\end{array}$ & $\begin{array}{l}\text { Sight-threatening } \\
\text { retinopathy }\end{array}$ \\
\hline Women/men (n) & $76 / 150$ & $37 / 59$ & $16 / 25$ \\
\hline Age (years) & $52.5 \pm 10.1$ & $56.7 \pm 10.4^{* *}$ & $57.2 \pm 8.2 *$ \\
\hline $\begin{array}{l}\text { Diabetes duration } \\
\text { (years) }\end{array}$ & $5.5 \pm 5.2$ & $12.6 \pm 7.3 * * *$ & $15.9 \pm 8.3 * * *$ \\
\hline BMI $\left(\mathrm{kg} / \mathrm{m}^{2}\right)$ & $27 \pm 5$ & $26 \pm 5$ & $29 \pm 5 * * \bullet \bullet$ \\
\hline $\mathrm{HbA}_{1 \mathrm{c}}(\%)$ & $7.9 \pm 1.6$ & $8.4 \pm 1.4$ & $8.9 \pm 1.5^{* * *}$ \\
\hline $\begin{array}{l}\text { Systolic BP } \\
(\mathrm{mmHg})\end{array}$ & $142 \pm 18$ & $153 \pm 23 * * *$ & $152 \pm 18 * * *$ \\
\hline $\begin{array}{l}\text { Diastolic BP } \\
(\mathrm{mmHg})\end{array}$ & $85 \pm 9$ & $85 \pm 8$ & $86 \pm 8$ \\
\hline $\begin{array}{l}\text { Urinary albumin } \\
\left(\mathrm{mgl}^{-1}\right)\end{array}$ & $0(0-3035)$ & $14(0-3110)$ & $42(0-4220) * * * \bullet \bullet \bullet$ \\
\hline $\begin{array}{l}\text { Serum creatinine } \\
\left(\mu \text { molx } 1^{-1}\right)\end{array}$ & $82(48-220)$ & $82(54-174)$ & $85(61-469)$ \\
\hline U-ACCR $\left(\times 10^{-3}\right)$ & $<0.01(<0.01-2.8)$ & $<0.01(<0.01-1.9)$ & $\begin{array}{l}0.01(<0.01-3.8) * * * \\
\bullet \bullet \bullet\end{array}$ \\
\hline
\end{tabular}

Values are given as mean \pm S.D. or as median (range).

$\mathrm{BP}=$ blood pressure; $\mathrm{U}_{-} \mathrm{ACCR}=$ albumin creatinine clearance ratio. $*=$ differences between no and background retinopathy, or no and sight-threatening Retinopathy. $\bullet=$ differences between background and sight-threatening. $*=\mathrm{P}<0.05, * *=\mathrm{P}<0.01, * * *=\mathrm{P}<0.001$

$\bullet=\mathrm{P}<0.05, \bullet \bullet=\mathrm{P}<0.01, \bullet \bullet \bullet=\mathrm{P}<0.001$. 
Table 2. Medical risk factors and mortality rate in patients with no retinopathy at baseline.

\begin{tabular}{|c|c|c|c|}
\hline & $\begin{array}{l}\text { No retinopathy } \\
(n=116)\end{array}$ & $\begin{array}{l}\text { Background } \\
(n=71)\end{array}$ & $\begin{array}{l}\text { Sight-threatening } \\
(\mathrm{n}=39)\end{array}$ \\
\hline Age at baseline (years) & $52 \pm 10$ & $54 \pm 12$ & $50 \pm 11$ \\
\hline $\begin{array}{l}\text { Duration at baseline } \\
\text { (years) }\end{array}$ & $4 \pm 5$ & $7 \pm 5 * *$ & $5 \pm 4$ \\
\hline $\mathrm{HbA}_{1 \mathrm{c}}(\%)$ & $7.3 \pm 1.3$ & $8.1 \pm 1.3 * * *$ & $9.1 \pm 0.9 * * * \bullet \bullet \bullet$ \\
\hline $\begin{array}{l}\text { Systolic BP } \\
(\mathrm{mmHg})\end{array}$ & $143 \pm 15$ & $147 \pm 15$ & $147 \pm 15$ \\
\hline Diastolic BP (mmHg) & $84 \pm 7$ & $84 \pm 6$ & $85 \pm 6$ \\
\hline $\begin{array}{l}\text { Urinary albumin }\left(\mathrm{mgl}^{-}\right. \\
\left.{ }^{1}\right)\end{array}$ & $33(10-4430)$ & $52(10-4670)$ & $90(0-4513) * * * \bullet$ \\
\hline $\begin{array}{l}\text { Serum creatinine } \\
\left(\mu \mathrm{mol} 1^{-1}\right)\end{array}$ & $112(58-878)$ & $133(60-979)$ & $92(59-852)$ \\
\hline Mortality(N) (\%) & $30(26 \%)$ & $18(25 \%)$ & $3(8 \%) * * \bullet \bullet$ \\
\hline
\end{tabular}

Values are given as mean \pm S.D. and as median (range).

$*=$ differences between patients with no retinopathy at follow-up and patients who had developed background or sight-threatening retinopathy, $\bullet=$ differences between patients who had developed background and patients who had developed sight-threatening retinopathy. * = $\mathrm{P}<0.05, * *=\mathrm{P}<0.01, * * *=\mathrm{P}<0.001$

$\bullet=\mathrm{P}<0.05, \bullet \bullet=\mathrm{P}<0.01, \bullet \bullet \bullet=\mathrm{P}<0.001$. 
Table 3. Underlying causes of death at follow-up in patients with no retinopathy at baseline.

\begin{tabular}{llll}
\hline & & Retinopathy degree at follow-up \\
\hline Mortality causes & No retinopathy & Background & Sight-threatening \\
& $(\mathrm{n}=116)$ & $(\mathrm{n}=71)$ & $(\mathrm{n}=39)$ \\
\hline Mortality $(\mathrm{N})(\%)$ & $30(26)$ & $18(25)$ & 1 \\
\hline AMI & 10 & 5 & 0 \\
Uremia & 0 & 2 & 0 \\
CVI & 2 & 1 & 1 \\
Other causes & 10 & 1 & 0 \\
Sudden death & 1 & 3 & 0 \\
Cancer & 5 & 2 & 1 \\
Heart failure & 2 & 4 &
\end{tabular}


Figure legends.

Figure 1.

The 10-year cumulative survival time according to retinopathy degree at baseline. Dotted line: no sight-threatening retinopathy. Full line: sight-threatening retinopathy.

\section{Figure 2}

The 10-year cumulative survival time in patients with no retinopathy at baseline. Dotted line: no sight-threatening retinopathy at follow-up. Full line: sight-threatening retinopathy at follow-up. 
Figure 1.

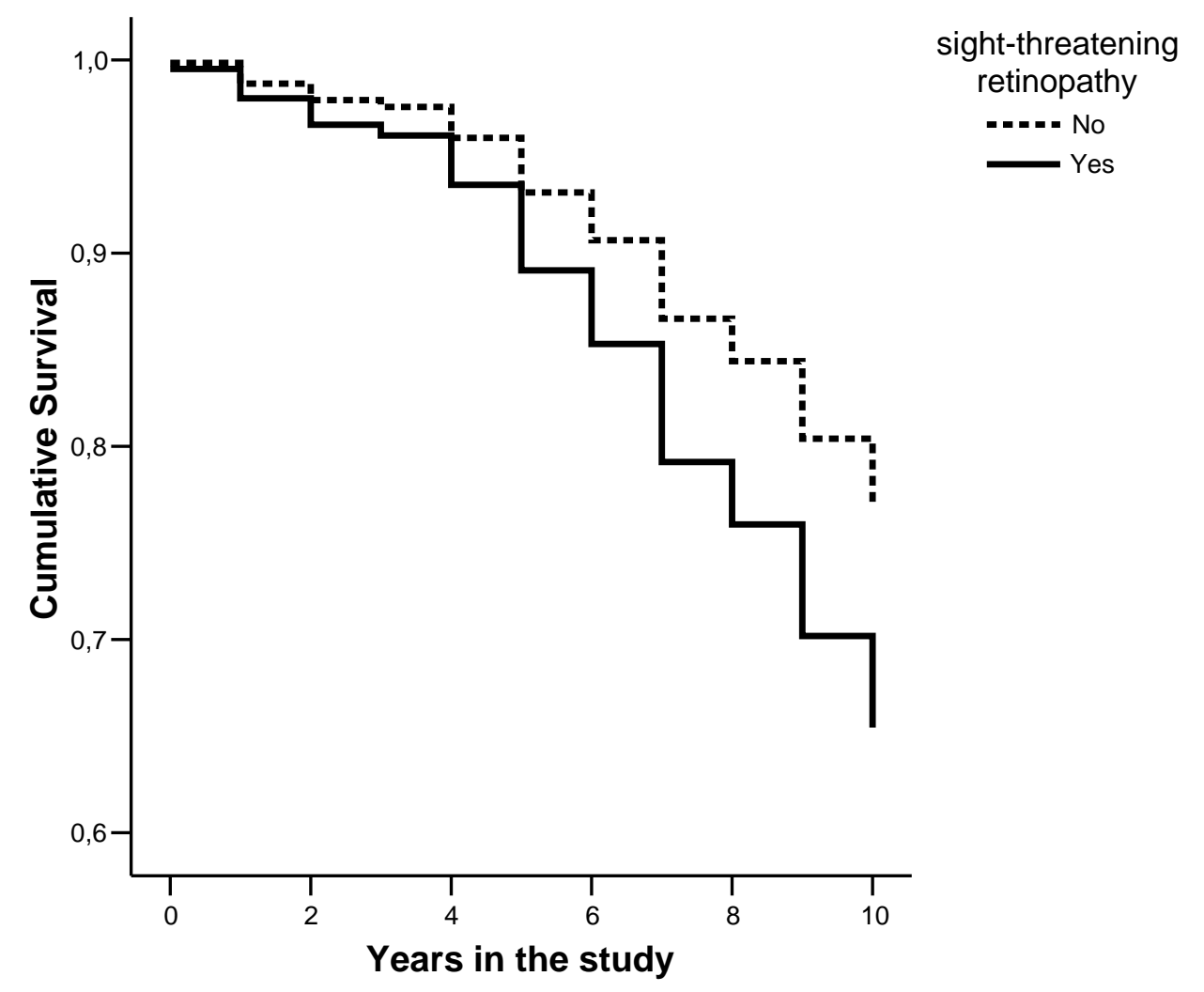


Figure 2.

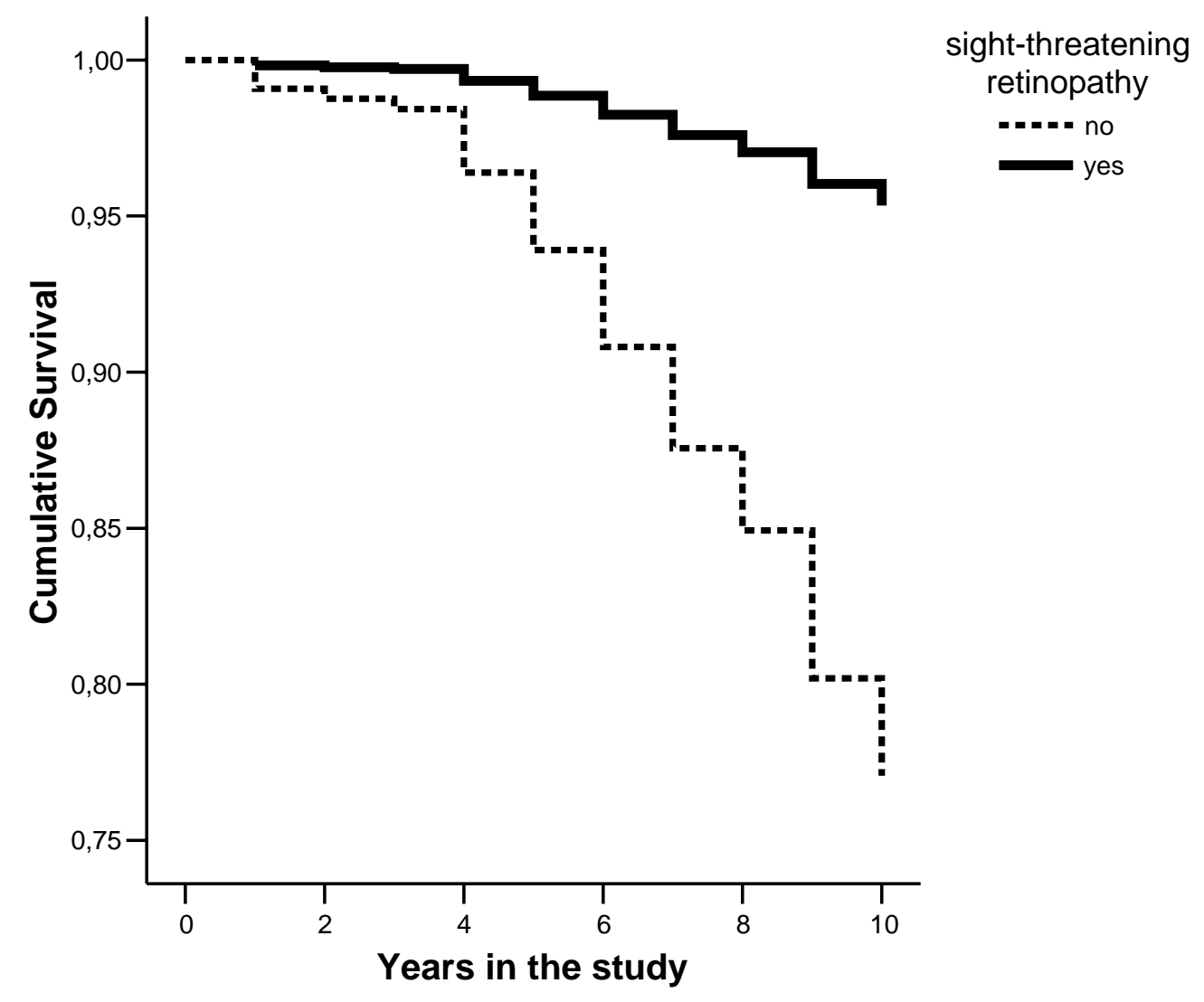


Attachment 1.

Survival Functions

No ACE treatment

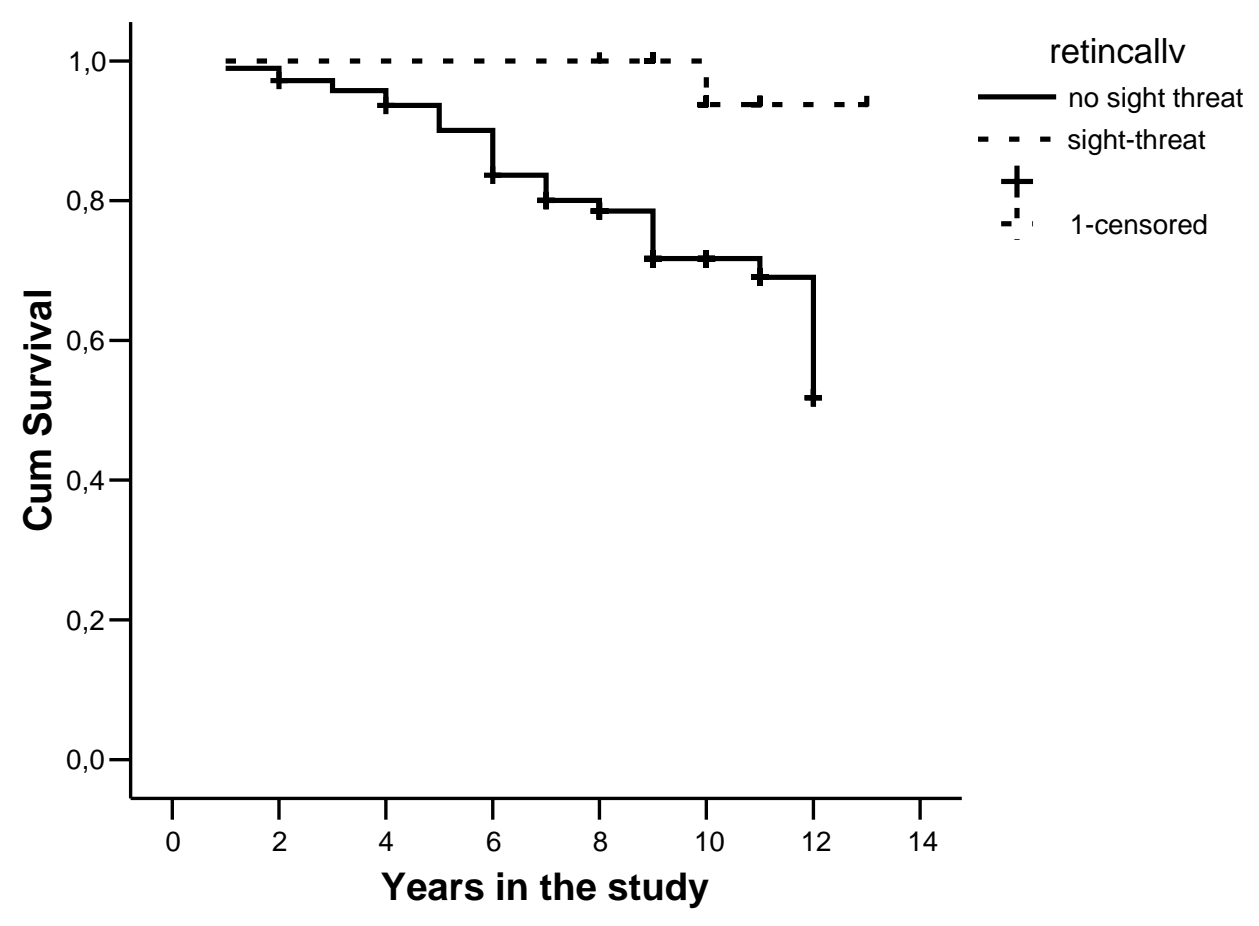


Attachment 2

Survival Functions

ACE Inhibitor treatment YEAS

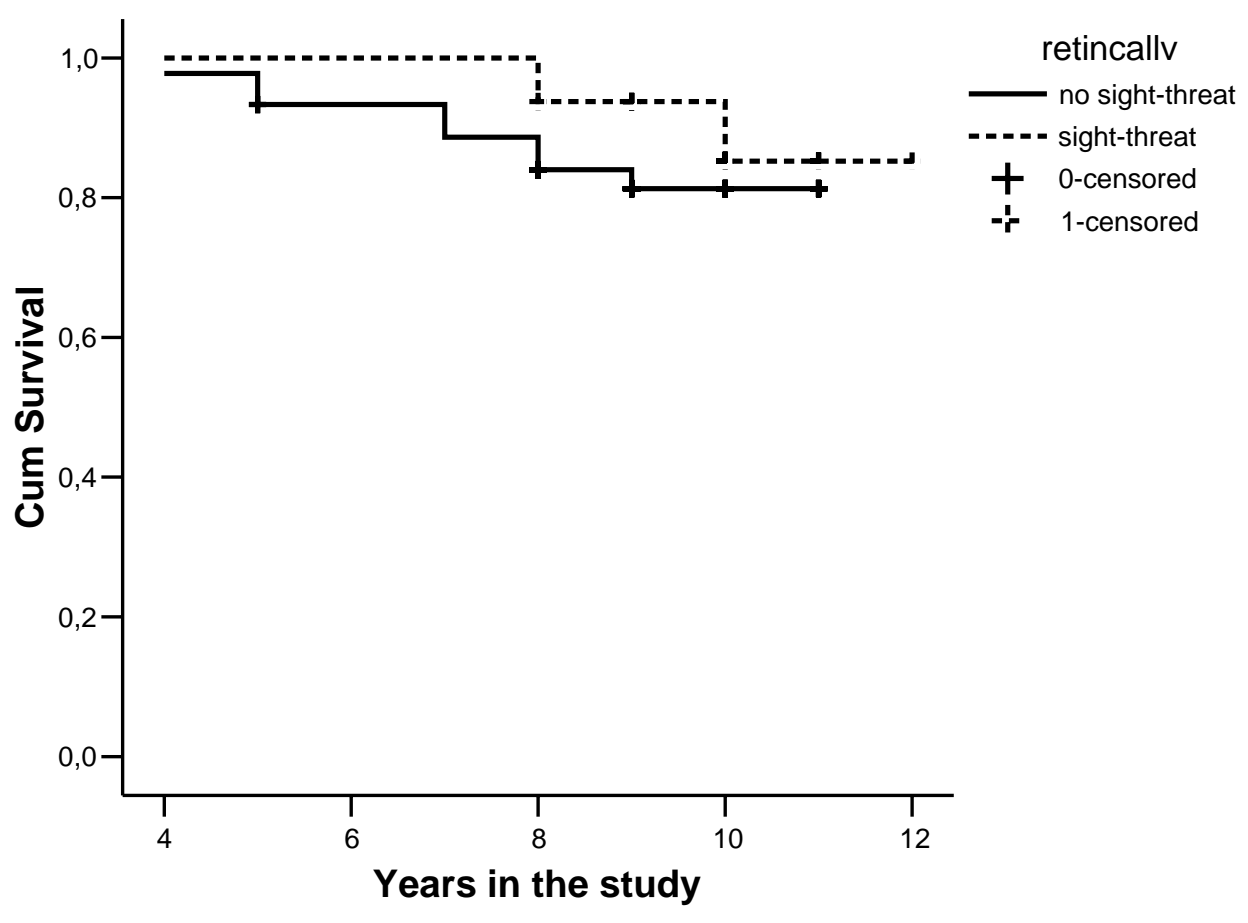

